\title{
The Design and Implementation of The E-BOOK Based on eye-movment
}

\author{
Lian $\mathrm{Li}^{1, \mathrm{a}}$, Shan Wang ${ }^{2, \mathrm{~b}}$ and Feilong Bai ${ }^{3, \mathrm{c}}$ \\ ${ }^{1}$ Anhui Xinhua University, Hefei, China \\ ${ }^{2}$ Anhui Xinhua University, Hefei, China \\ ${ }^{3}$ Anhui Xinhua University, Hefei, China \\ ahfmuyi_0421@163.com, b2595369779@qq.com, c875498813@qq.com
}

Keywords: EOG, Acquisition, Identification, E-book.

\begin{abstract}
The thesis researches and designs one system based on eye movement EOG, the system contains three modules. They are signal acquisition module, EOG identification module and control making module. The main works are eye movement pattern recognition with three kinds of EOG, which are continuous blink EOG, Up-look EOG and Right-look EOG. With the result of the second section, an EOG pattern would be confirmed and turned into a control instruction for E-book system. After experiments, the system had the validity and stability.
\end{abstract}

\section{Introduction}

\section{HCI status and significance}

HCI is the interaction between human and computer, which the concept proposed in the last century fifty's. Now it is still a popular major. HCI design a bridge between product and user, this bridge let the user know how to operate the product, such as a radio play, mobile GPS, mobile phone interface, even in the fatigue driving detection and so on. These are HCI professionals' learning direction and future focus.

With the development of science and technology is increasingly integrated into people's lives, the computer is not only engineering and scientific tools, but also began to be recognized as an important part of solutions to major social problems in health care, education, energy and environmental protection. In the future of "the senses world", HCI will be increasingly applied to the future and influence people's life.

\section{Project introduction}

In this project, an E-BOOK system based on $\mathrm{VC}++$ was designed and implemented with different eye-moving control means. The control measures include the energy of the eye-movement, the direction of eye-movement and blinks. The controls are e-books' selecting and page turning.

In this thesis, there are three steps to use the system successfully by signal acquisition, EOG identification and control making. The thesis is organized in five sections. Section two presents signal acquisition module about BCI (Brain Computer Interfaces, BCI), Section three describes EOG identification module with identification algorithm. Section four presents control making module, and finally section is conclusion. 


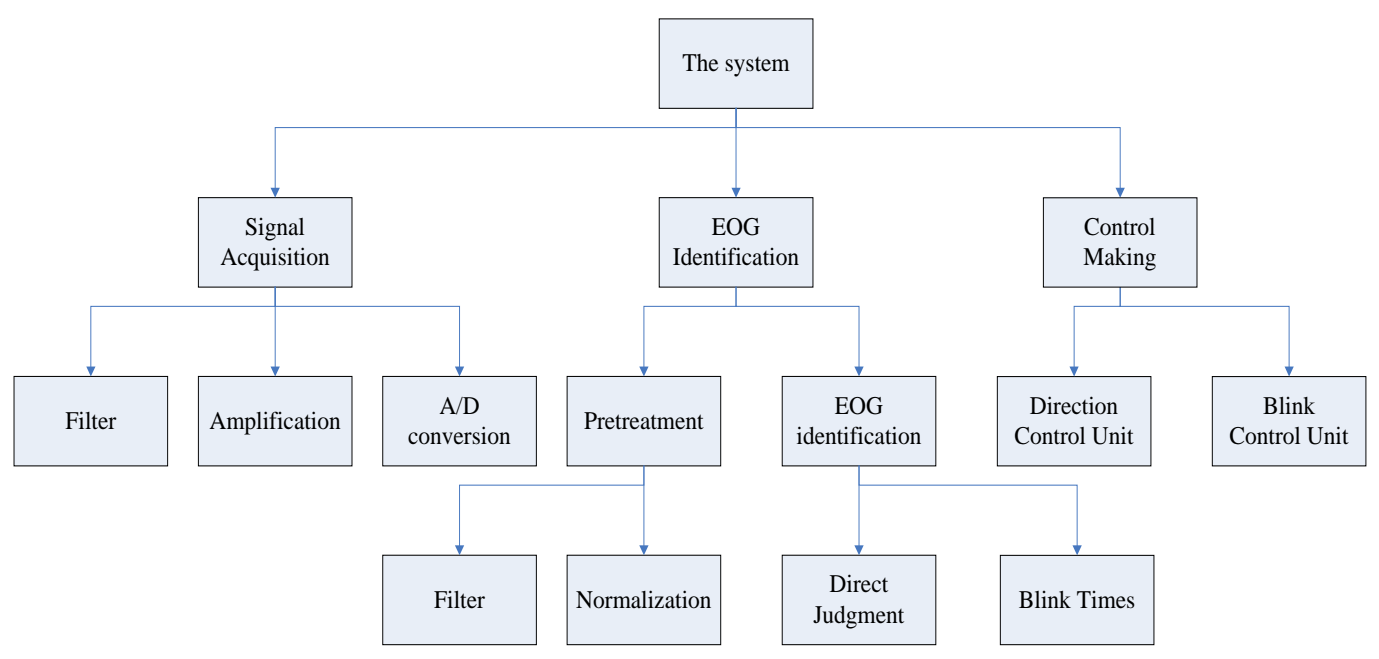

Fig.1 The system module architecture chart.

\section{Signal Acquisition Module}

The Acquisition Equipment In this thesis, one based on Bluetooth acquisition equipment was taken as signal acquisition module's core with a Bluetooth dongle. Via the application of a single electrode and signal-processing unit in a headband arrangement, the equipment's sampling rate is $512 \mathrm{HZ}$, hardware filtering is $3 \mathrm{HZ}$ to $100 \mathrm{HZ}$, and its baud rate is 57600 . The core's features are directly connects to dry electrode, one EOG channel with three contracts: EOG electrode located above the left eye on the forehead; reference and ground electrodes located on two earlobes. In order to filter noise, the core can detect abnormal state of poor contract and filter out 50/60 $\mathrm{HZ}$ ac interference.

The Bluetooth device has to be wore well, the three electrodes has to adjoin the right point just like the show in figure 2. Otherwise, it would make mistakes produced by electrodes attached instability.

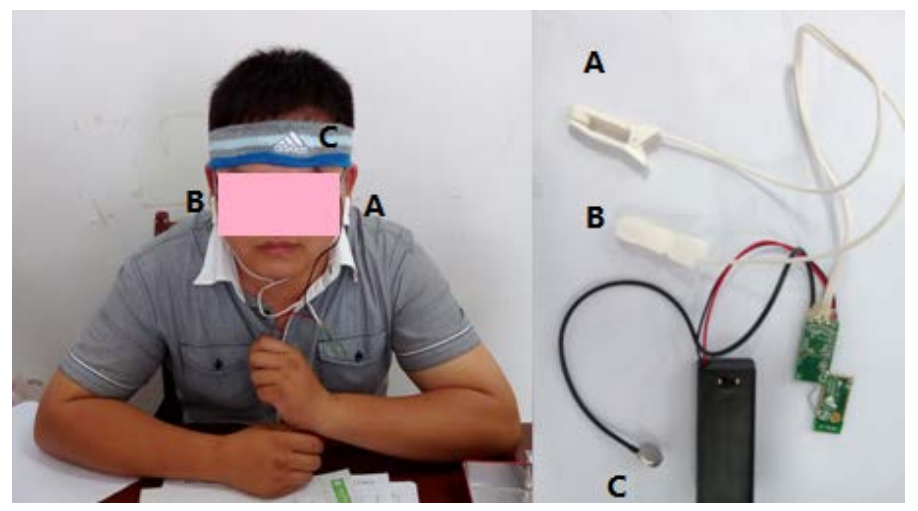

Fig.2 The acquisition equipment electrodes match chart.

EOG Identification Module. Do not print page numbers: Please number each sheet toward the middle near the bottom (outside the typing area) with a soft pencil.

Pretreatment. EOG signal frequency range is between $0.1 \mathrm{~Hz}$ and $38 \mathrm{~Hz}$. The main information is contained in $10 \mathrm{~Hz}$ below. On the basis of the collected EOG, after hardware circuit filter of EOG signals is not completely pure, still contains some background noise. On the basis of hardware filtering, in order to further eliminate the interference caused by users' blink and eyeball tremor. In order to keep the main EOG information, we have to design digital filter. This thesis chooses a cut-off frequency of three-pole $7 \mathrm{~Hz}$ Butterworth low-pass filter and a cut-off frequency of three-pole $0.1 \mathrm{~Hz}$ Butterworth high-pass filter compose of a band-pass filter for filtering EOG signal. The original signal and the filtered signal got by the above filter are shown in the figure 3 . 


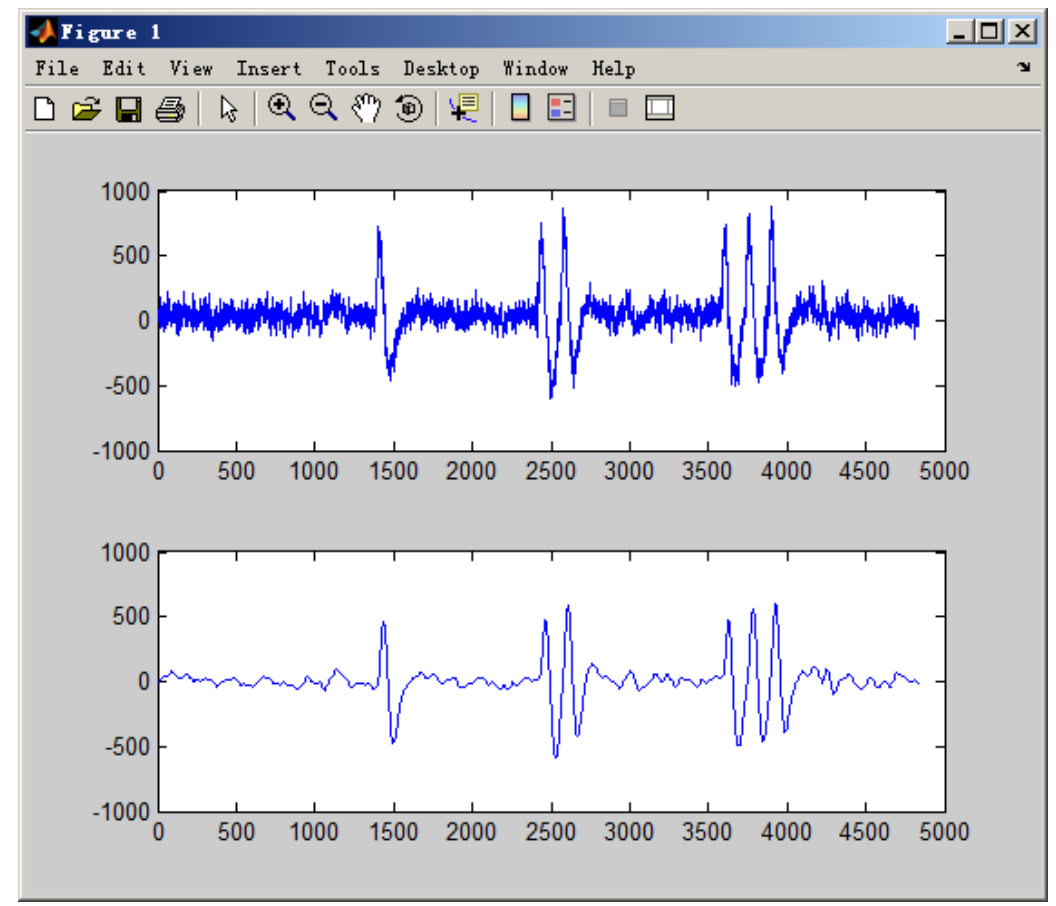

Fig.3 Original signal's waveform and filtered signal's waveform.

In addition to the blink of eye movement patterns, in this thesis, the other two eye movement patterns were taken, which are Up-look and Right-look. The Up-look EOG is shown in the following figure's left part, and the Right EOG is shown in the following figure's right part. The difference of the blink EOG, Up-look EOG and Right-look EOG are wave shape and energy. The energy of the blink EOG is maximal. The Right-look EOG waveform is like sine wave. The Up-look EOG waveform is consists of two different sizes of sine wave, and the second one's energy is twice the front one.
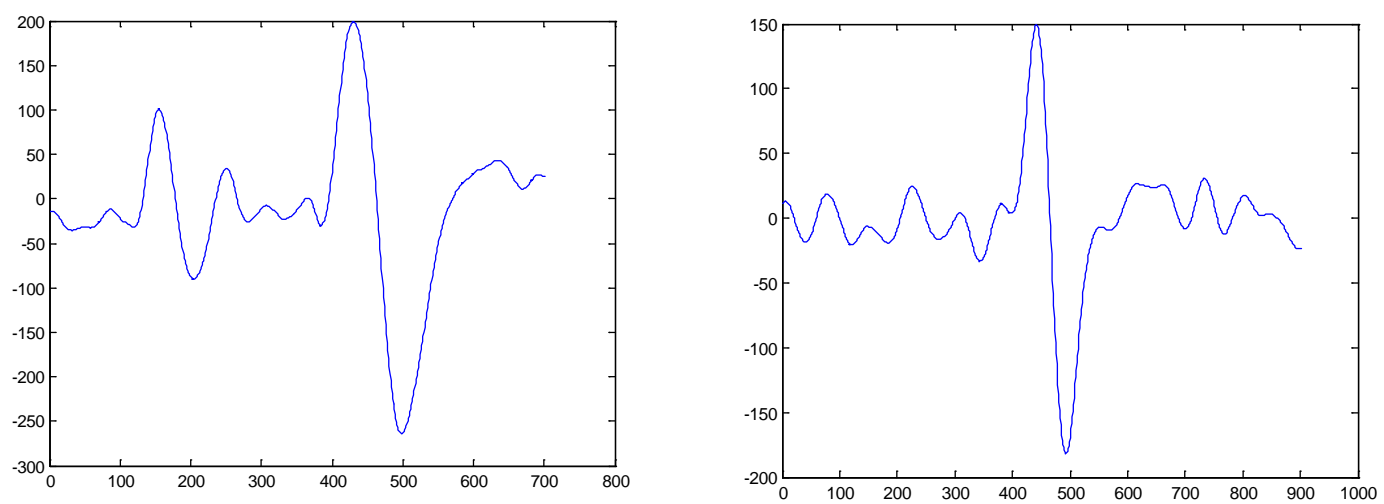

Fig.4 the filtered Up-look EOG(left) and Right-look EOG (right) waveform

EOG identification. The unit describes the solution of determining direction of looking. The steps are as follows:

- Determine the data processing frequency with 512 data after filtering.

- When the data is greater than the direction critical value for judging Up-look’s first half, create a new array to record the next 200 data. If the maximum is between 100 and 200, the movement is UP-look or Right-look, else is blink or noting.

- if the maximum is between 100 and 200, record the value's subscript. And then judge the next similar array. If it exists, calculate the difference of the two subscripts. If the difference is smaller than a specific value, a Up-look EOG is determined, else is Right-look EOG.

Note that the above parameters can be manually adjusted. 
This thesis identifying the blinking movements and accumulating the continuous blink in the light of EOG waveform features. In figure 5, one blink movements could be mainly identified by seven kinds of EOG characteristics. In this paper, mid-split sites, crest value and the distance between two crests are chosen to identify blink and the continuous blink times

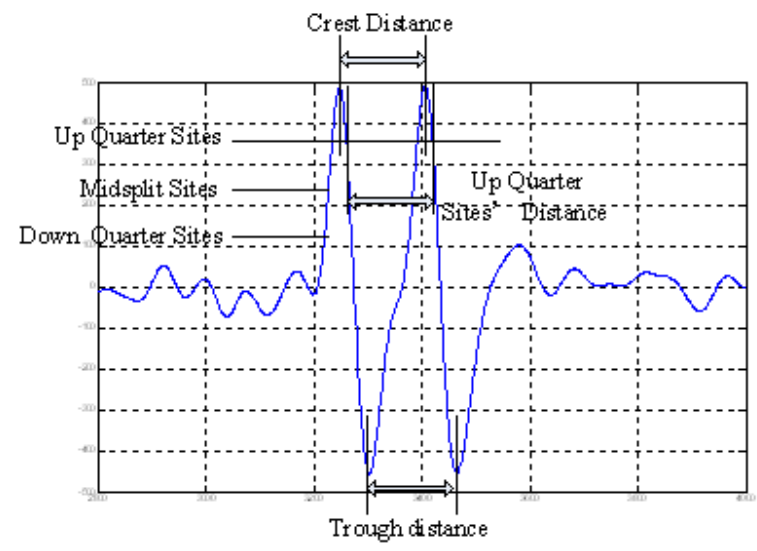

Fig. 5 the features of the continuous blink

Control Making Module. In this thesis, the control object is an E-Book reading system implemented based on MFC. In this project, user could select any notepad files from a book list and read forward or back without hand moving. Two groups control models are made for the target. Group one, eyeblink counts got in 3.2.2 turn to the book selecting operations, as blink twice stand for selecting, and the next blinks range from 1to 6 stand for the first and the sixth item books in the list. Group two, eye movement directions got in 3.2.1 turn to reading controls, as looking up stand for reading back, and looking right stand for reading forward. In the second group, a thrice blinking stands for pause reading, the next thrice blinking again means restart reading.

Conclusion. The thesis main works are signal acquisition, different EOG identification and the control make. 10 subjects were invited to participate in the test. The male and the female is half of each. Each person wore the equipment well, and then, chose a book from the book list by blink. After selected, the subjects were asked to turning page with looking up or right. After experiments, the system had the validity and stability

\section{References}

[1] ZHAO L, WU X P, LI M, et al. Implementation of the EOG-Based Human Computer Interface System[c]. Bioinformatics and Biomedical Engineering, the $2^{\text {nd }}$ international Conference, 2008.2188-2191.

[2] Q. Ding, K. Tong, Guang Li. Development of an EOG(Electro-Oculography) Based Human-Computer Interface[C]. Proceedings of the 2005 IEEE EMBS 27th Annual Conference, Shanghai, China, Sept.1-4,2005:6829-6831.

[3] Hori.J, Sakano.K, Saitoh.Y. Development of Communication Supporting Device Controlled by Eye Movements and Voluntary Eye Blink[C]. Proceedings of the 26th Annual International Conference of the IEEE EMBS, San Francisco, CA, USA,2004:4302-4305.

[4] Grandhi, S. Jones, Q. Technology-mediated interruption management. International Journal of Human-Computer Studies, 68(5), 2010:288-306.

[5] McGrew, K.S. CHC theory and the human cognitive abilities project: Standing on the shoulders of the giants of psychometric intelligence research. Intelligence, 37, 2009:1-10 